\title{
2. Survival impact of HER-2/Neu, Cox-2, urokinase plasminogen activator (upa), cytokeratin 17/5,6 and other markers with long-term outcome of early breast cancer. Report from the British Columbia Tissue Micro-Array Project (BCTMAP)
}

J. Ragaz ${ }^{1}$, M. Lippman², M. Van Rijn ${ }^{3}$, A. Brodie ${ }^{4}$, Danijela Jelovac ${ }^{4}$, T. Nielsen ${ }^{1}$, S. Dedhar, D. Huntsman ${ }^{1}$, M. Hayes ${ }^{1}$, S. Dunn ${ }^{1}$, M. Cheung ${ }^{1}$, G. Sledge ${ }^{5}$, S. Chia ${ }^{1}$, A. Harris ${ }^{6}$, C. Bajdik ${ }^{1}$, C. Speers ${ }^{1}$, J. Spinelli ${ }^{1}$, and D. Hayes ${ }^{2}$

${ }^{1}$ University of British Columbia, Vancouver, Canada; ${ }^{2}$ University of Michigan, Ann Arbor, MI; ${ }^{3}$ Stanford University, Stanford, CA; ${ }^{4}$ University of Maryland, Baltimore, MD; ${ }^{5}$ University of Indiana, Indianapolois, IN, USA; ${ }^{6}$ University of Oxford, $U K$

Tumor samples are available from over 19,600 Stage I-III breast cancer patients treated according to evolving British Columbia guidelines from 1978 to 1990 . A tissue mico-array (TMA) was constructed from 930 of these patients, all of whom participated in randomized or phase II studies. Outcome was defined as 20-year Breast Cancer specific Survival (BrCaSS), with events defined as Breast Ca death. Follow up was median 17.8 years (ranges 11-28). Multiple tumor markers were tested, and results correlated with 20-year BrCaSS for markers expressed versus non-expressed.

\begin{tabular}{lllr} 
20-year BrCaSS & & & \\
\hline Marker & Expressed (\%) & Not expressed (\%) & $p$-Value \\
\hline Her-2-IHC & 34 & 48 & $<0.0001$ \\
Her-2 FISH & 33 & 48 & 0.0004 \\
Her-2 ACIS IHC** & 32 & 47 & 0.0001 \\
Cytokeratins 17/5,6 IHC: & 35 & 47 & $<0.0020$ \\
uPA IHC & 46 & 52 & 0.0300 \\
Cox-2 IHC & 32 & 45 & 0.0260
\end{tabular}

No difference in BrCaSS was found for aromatase, integrin-linked kinase (ILK), IGF-1 and Topo-isomerase-2. The negative predictive value of IHC versus FISH and ACIS-IHC versus FISH was 96 and 97\%, respectively. The positive predictive value of IHC versus FISH and ACIS-IHC versus FISH was 84 and $84 \%$, respectively. All tests, with the exception of HER-2 FISH were done by IHC. Results of other markers (VEGF, ER/PgR, hypoxia markers, etc.), and an interactive multivariate analysis adjusting for conventional prognostic factors and for all above markers, are in progress. Conclusions 1. The TMA is a technique which provides opportunity for rapid screening of multiple genetic markers. 2. Expression of Her-2/Neu, uPA, Cox-2 and Cytokeratin 17/5,6 (but not of Aromatase, ILK, TOPO-II and IGF-1) is associated with inferior BrCaSS. 3. HER-2 determination by ACIS-IHC provides comparable results to IHC done manually (with a potential for more uniform reporting), and both provide comparable results to Her-2 assessment by FISH. ${ }^{* *}$ ACIS-IHC:IHC red by Automated Cell Image System (M.L.) 